\title{
Der Mindestlohn von 12 Euro kommt - die sozialpolitischen Risiken bleiben
}

Aktuell beträgt der Mindestlohn 9,60 Euro pro Stunde. Bereits beschlossen ist, den Mindestlohn bis zum 1. Juli 2022 in zwei Stufen um knapp $9 \%$ auf 10,45 Euro anzuheben. Damit will sich die zukünftige Ampelkoalition jedoch nicht begnügen. Sie hat sich in ihrem Sondierungspapier darauf geeinigt, die Wahlversprechen von SPD und Grünen umzusetzen und den Mindestlohn im Rahmen einer einmaligen Anpassung im kommenden Jahr auf zwölf Euro pro Stunde zu erhöhen - das entspricht einer zusätzlichen Erhöhung des Mindestlohns um rund $15 \%$. In der Summe erhöht sich damit im kommenden Jahr der Mindestlohn um $25 \%$ gegenüber dem Jahr 2021.

Was ist davon zu halten? Zunächst einmal: Beim Mindestlohn erleben wir dasselbe wie zuvor schon in anderen Politikbereichen, z. B. bei der Rentenpolitik oder der Diskussion um die Aufweichung der Schuldenbremse. Die deutsche Politik verabschiedet sich immer mehr von der Bindung an feste Regeln, die die langfristige Tragfähigkeit des Wirtschafts- und Sozialsystems vor der Verlockung populärer, aber bestenfalls kurzfristig erfolgreicher Maßnahmen schützen sollen und die zumindest in Ansätzen ökonomischen Kriterien Rechnung tragen. Die Umsetzung von Wahlversprechen ersetzt langfristig tragfähige Politik. 2015 wurde mit der Einführung des Mindestlohns die Mindestlohnkommission geschaffen. Sie soll den Mindestlohn so anpassen, dass auch bei sich verändernden Rahmenbedingungen ein angemessener Mindestschutz der Beschäftigten gewährleistet wird. Als gleichberechtigtes Kriterium bei dieser Entscheidung über die Höhe des Mindestlohns schreibt das Gesetz zudem vor, dass die Beschäftigung nicht gefährdet werden soll. Der Spielraum der Mindestlohnkommission ist dabei sehr beschränkt, denn sie hat sich "nachlaufend an der Tarifentwicklung" zu orientieren (§ 9 Abs. 2 MiLoG). Darauf gründet sich die ursprünglich geplante Erhöhung des Mindestlohns auf 10,45 Euro.

Das aber ist der kommenden Regierung viel zu wenig. Die zukünftige Ampelkoalition will sicherstellen, dass - wie die SPD es im Wahlkampf forderte - die Geringverdienenden den Anschluss an die Mitte halten. Das sei aus ihrer Sicht machbar, denn die Evaluierung des Mindestlohngesetzes hätte Nachbesserungsbedarf aufgezeigt und

(C) Der/die Autor:in 2021. Open Access: Dieser Artikel wird unter der Creative Commons Namensnennung 4.0 International Lizenz veröffentlicht (creativecommons.org/licenses/by/4.0/deed.de).

Open Access wird durch die ZBW - Leibniz-Informationszentrum Wirtschaft gefördert. zudem bewiesen, dass der Mindestlohn der deutschen Volkswirtschaft nicht geschadet hat.

\section{Empirische Evidenz zum Mindestlohn}

Der dritte Bericht der Mindestlohnkommission fasst die vorliegende empirische Evidenz aus den diversen von der Mindestlohnkommission in Auftrag gegebenen Evaluierungsprojekten wie folgt zusammen: Durch die Einführung des gesetzlichen Mindestlohns im Jahr 2015 sind die Bruttostundenlöhne am unteren Rand der Lohnverteilung deutlich stärker gestiegen als die Löhne in nicht betroffenen Beschäftigungsverhältnissen. Die Einführung des Mindestlohns hat also Wirkung gezeigt, und die Mindestlohnkommission hat dafür gesorgt, dass Mindestlohnempfangende in den Folgejahren an der allgemeinen Lohnentwicklung sogar überproportional partizipieren konnten. Einen Nachbesserungsbedarf kann man daraus jedenfalls nicht ableiten.

Was für die Stundenlöhne gilt, trifft jedoch nicht im gleichen Maße auf die Bruttomonatseinkommen zu. Bezieht man Arbeitszeitanpassungen mit ein, zeigt sich kein so klares Bild. Studien, die auf von Arbeitgebenden berichtete Daten zu Löhnen und Arbeitszeiten zurückgreifen (z. B. die Verdienststrukturerhebung oder das IAB-Betriebspanel), finden in der Regel positive Effekte des Mindestlohns auf die Monatslöhne der Betroffenen, die aber im Durchschnitt kleiner ausfallen als die Wirkung auf die Stundenlöhne. Hinzu kommen allerdings noch Einkommens-

Prof. Dr. Andreas Knabe ist Professor für Finanzwissenschaft an der Otto-von-GuerickeUniversität Magdeburg.

Prof. Dr. Ronnie Schöb ist Professor für Internationale Finanzwissenschaft an der Freien Universität Berlin.

Prof. Dr. Marcel Thum ist Professor für Finanzwissenschaft an der Technischen Universität Dresden und Direktor des ifo Instituts in Dresden. 
zuwächse, die sich aus dem Wechsel von Beschäftigten von kleineren Firmen mit niedrigen Löhnen zu größeren, produktiveren Firmen mit höheren Löhnen ergaben (Dustmann et al., 2021). In Arbeitnehmerbefragungen, wie dem Sozioökonomischen Panel (SOEP), können überhaupt keine Effekte auf die Monatseinkommen nachgewiesen werden. Ursächlich für die unterschiedlichen Wirkungen auf Stunden- und Monatslöhne ist vor allem ein Rückgang der Wochenarbeitszeit der betroffenen Beschäftigten (Mindestlohnkommission, 2020, 49 f.). Auch wenn die Lohnstruktur durch den Mindestlohn im unteren Bereich gestaucht wurde und die Ungleichheit der Stundenlöhne abgenommen hat, so ist der Anteil der Beschäftigten im Niedriglohnsektor doch nur leicht zurückgegangen. Die Zahl der auf aufstockende Alg-II-Leistungen angewiesenen Beschäftigten hat sich leicht reduziert, wobei dieser Rückgang zum Teil auf Einkommenssteigerungen, zum Teil aber auch auf Beschäftigungsverluste zurückzuführen ist. Darüber hinaus sei keine klare Wirkung des Mindestlohns auf die Armutsgefährdungsquote feststellbar (Mindestlohnkommission, 2020, 76 ff.).

\section{Warum bleiben die Erfolge des Mindestlohns aus?}

Auch wenn noch viele Unklarheiten bestehen, so könnte man aus letzteren Befunden schlussfolgern, dass die Einführung des Mindestlohns es bislang nicht geschafft hat, den Anschluss der Geringverdienenden an die Mitte wiederherzustellen. Keinesfalls zwangsläufig lässt sich daraus aber der Umkehrschluss ziehen, dass der Mindestlohn bislang nur viel zu gering war und eine deutliche Erhöhung um $25 \%$ auf 12 Euro pro Stunde jetzt den gewünschten Erfolg mit sich bringen würde. Stattdessen ist es wichtig, sich die Gründe für das Ausbleiben der von den Befürworter:innen des Mindestlohns erhofften Erfolge vor Augen zu halten.

Ein Grund ist im Grundsicherungssystem unseres Sozialstaates zu finden. Die Grundsicherung stockt Arbeitseinkommen auf, wenn dieses allein kein existenzsicherndes Haushaltseinkommen sichert. In der Begründung des Mindestlohngesetzes wurde seinerzeit auf einen vollzeitbeschäftigten Alleinstehenden abgestellt, der durch den Mindestlohn in die Lage versetzt werden sollte, sein Existenzminimum selbst zu verdienen. Für diese Gruppe mag der Mindestlohn dafür auch ausreichend hoch sein. Allerdings sind nur etwa $3 \%$ der aufstockenden Alg-IIBeziehenden alleinstehende Vollzeitbeschäftigte. Bei allen anderen führen eine geringere Zahl an Arbeitsstunden oder Bedarfe weiterer Haushaltsmitglieder dazu, dass sie selbst mit dem Mindestlohn weiterhin auf das Alg II angewiesen sind. Für die meisten aufstockenden Alg-IIEmpfänger:innen bedeutet eine Erhöhung des Mindestlohns primär, dass das höhere Einkommen angerechnet und staatliche Transfers zurückgefahren werden. Die Betroffenen profitieren, wenn überhaupt, nur im geringen Maße von der Erhöhung des Mindestlohns.

Es wird gerne übersehen, dass Vollzeitbeschäftigte, die 2018 einen Stundenlohn oberhalb des Mindestlohns aber unterhalb von zehn Euro erhielten, nur zu $13 \%$ als einkommensarm galten - das liegt um vier Prozentpunkte unter dem Durchschnitt der Bevölkerung. Das liegt daran, dass die Armutsgefährdung viel stärker vom Beschäftigungsumfang als von der Höhe des Mindestlohns abhängt. Unter Teilzeitbeschäftigten mit gleichem Stundenlohn lag der Anteil der Einkommensarmut mit $26 \%$ fast doppelt so hoch wie bei den Vollzeitbeschäftigten (Schröder und Kestermann, 2020). Es ist also auch im Hinblick auf das Ziel, die Geringverdienenden näher an die Mitte zu bringen, unerlässlich, neben den Stundenlöhnen auch die Arbeitszeiten zu berücksichtigen und sich genau anzuschauen, welche Wirkungen der Mindestlohn auf beide Größen hat.

Bezüglich der Beschäftigung deuten die vorliegenden Evaluationsstudien darauf hin, dass der Mindestlohn die Zahl geringfügiger Beschäftigungsverhältnisse deutlich reduziert hat. Bei den sozialversicherungspflichtigen Beschäftigungsverhältnissen sind hingegen keine klaren Effekte festzustellen. Die vorliegenden Studien spannen ein Intervall an geschätzten Beschäftigungseffekten auf, das von einem Zugewinn an 11.000 bis zu einem Verlust von 200.000 Stellen reicht. In der Gesamtschau deuten diese Studien darauf hin, dass die Gesamtzahl an Beschäftigungsverhältnissen durch den Mindestlohn sowohl kurzals auch mittelfristig leicht zurückgegangen ist (Mindestlohnkommission, 2020, 100 ff.).

Zusätzlich zu den geschätzten Verlusten von Stellen ist aber auch noch die Veränderung der Arbeitszeit in bestehenden Arbeitsverhältnissen zu betrachten. Es gibt zahlreiche Hinweise, dass Unternehmen, z. B. mit einer Verkürzung der Öffnungszeiten oder mit Arbeitsverdichtung, auf den Mindestlohn reagiert haben. Evaluationsstudien zeigen, dass unmittelbar nach Einführung des Mindestlohns die Arbeitszeit betroffener Arbeitnehmer:innen spürbar reduziert wurde. Burauel et al. (2020) berichten von einem Rückgang der vertraglich vereinbarten Arbeitszeit von sozialversicherungspflichtig Beschäftigten um $5 \%$. Allerdings zeigen sie auch, dass die tatsächlich geleistete Arbeitszeit weniger stark gesunken war, was auf ein illegales Unterlaufen des Mindestlohns hindeuten könnte. Volkswirtschaftlich errechnet sich daraus ein Rückgang um 79.000 Vollzeitäquivalente bzw. um 92.000 Stellen, wenn man die tatsächliche Verteilung auf Vollzeit- und Teilzeitbeschäftigte zugrunde legt. Caliendo et al. (2017) ermitteln einen mindestlohninduzierten Rückgang der vertraglich vereinbarten Arbeitszeit der 
Arbeitnehmer:innen im unteren Lohnquintil von 3,5\%, wobei die tatsächliche Arbeitszeit in ähnlicher Größenordnung zurückgeht. Rechnet man diesen Rückgang der Arbeitszeit entsprechend um, ergibt sich über sozialversicherungspflichtig und geringfügig Beschäftigte hinweg ein Verlust von insgesamt 140.000 Stellenäquivalenten. Für die Folgejahre nach 2015 ist die Studienlage uneinheitlich, deutet aber auf eine schwach negative Wirkung des Mindestlohns auf das Arbeitsvolumen hin (Mindestlohnkommission, 2020, 13 und 116).

\section{Wie der Mindestlohn unterlaufen wird}

Der gesetzliche Mindestlohn beeinflusst die Beschäftigung nur dann, wenn er auch tatsächlich bezahlt wird. Allerdings wird der Mindestlohn offenbar häufig unterlaufen. Hierfür werden verschiedene Praktiken genutzt. Diese umfassen z.B. die unkorrekte Erfassung der Arbeitszeit, die Nichtvergütung von Vor- und Nacharbeiten, die Vereinbarung von Stücklöhnen mit unrealistisch hohen Zielvorgaben, die unzulässige Anrechnung von Kost und Logis oder die ungerechtfertigte Anwendung von Ausnahmeregeln für Praktikant:innen oder Scheinselbstständigkeit (Mindestlohnkommission, 2020, 65). Laut der Verdiensterhebung 2019, einer Arbeitgeberbefragung, erhielten 1,4 Mio. Arbeitnehmer:innen den Mindestlohn, mehr als eine halbe Million erhielten aber auch 2019 noch weniger als den Mindestlohn (Statistisches Bundesamt, 2020). Die Berechnungen des DIW auf Grundlage der Arbeitnehmerbefragung im SOEP 2018 liefern sogar deutlich höhere Zahlen. Sie geben die Zahl der anspruchsberechtigten Beschäftigten mit berechneten vereinbarten Stundenlöhnen unterhalb des gesetzlichen Mindestlohns für 2018 mit 2,4 Mio. (6,8\%) an. Legt man die berechneten tatsächlichen Stundenlöhne zugrunde, liegt die Quote noch höher (Fedorets et al., 2020).

Das lässt in doppelter Weise an den Vorteilen des Mindestlohns zweifeln. Erstens erreicht der Mindestlohn viele Betroffene gar nicht. Wenn eine Anhebung auf 12 Euro den Anschluss einiger Geringverdienender an die Mitte sicherstellt, bedeutet das zugleich, dass er einen beträchtlichen Teil der Angestellten noch weiter ins Abseits stellt. Wenn man jedoch für eine bessere Durchsetzung des Mindestlohns sorgt, sind zweitens die Gefahren für die Beschäftigung ungleich höher als die bisher beobachteten Effekte. Der Mindestlohn hilft Angestellten nicht dabei, den Anschluss zur Mitte zur halten, wenn sie zugleich von starken Arbeitszeitreduktionen getroffen werden.

\section{Internationale Erfahrungen uneinheitlich}

Schaut man auf die internationalen Erfahrungen mit dem Mindestlohn, auf die ja auch von Befürwortenden des Mindestlohns regelmäßig verwiesen wird, so zeigt sich dort ein vielschichtiges, nicht leicht zu interpretierendes Bild. Daraus wird mitunter der Schluss gezogen, dass negative Beschäftigungseffekte eher unwahrscheinlich sind (hierzu etwa Manning, 2021). Aber lack of evidence ist eben nicht evidence of absence - eine Unterscheidung, die gerade, wenn es um sozialpolitische Folgen geht, unbedingt beachtet werden sollte. Befragt man die Autor:innen der empirischen Studien zu den Beschäftigungseffekten des Mindestlohns nach der von ihnen am meisten präferierten Schätzung, so zeigt sich zumindest eine deutliche Tendenz (Neumark und Shirley, 2021). Die Befragung der Autor:innen von insgesamt 69 Studien zum Mindestlohn in den USA (auf die ja gerne in der deutschen Diskussion verwiesen wird) ergab das folgende Ergebnis: 78,9\% weisen negative Beschäftigungseffekte auf, 46,1\% sind negativ und auf dem $5 \%-\mathrm{Ni}-$ veau signifikant. Nur in 3,9\% der Fälle wurden positive, auf dem $5 \%$-Niveau signifikante Beschäftigungseffekte festgestellt. Insbesondere junge Erwachsene und Menschen mit geringerem Bildungsniveau sind stärker von negativen Beschäftigungseffekten betroffen. Diese Interpretation der vorliegenden Studien deutet daher darauf hin, dass Mindestlöhne tendenziell negative Beschäftigungswirkungen haben. Auf keinen Fall sollte die Studienlage als Beweis dafür interpretiert werden, dass Mindestlöhne für die Beschäftigung unschädlich seien.

Der Mindestlohn scheint in Deutschland einen guten Start gehabt zu haben. Bei seiner Einführung befand sich der Arbeitsmarkt in Deutschland in einer starken und langanhaltenden Aufschwungphase. Der Mindestlohn hat die Fortsetzung dieses Aufschwungs nicht merklich gestört. Daraus erklärt sich auch die große Zustimmung, die eine weitere Erhöhung des Mindestlohns in der Öffentlichkeit erfährt. Die Berichte der Mindestlohnkommission und die in ihnen berücksichtigten wissenschaftlichen Studien zeigen allerdings, dass der Mindestlohn nicht spurlos an der Beschäftigung vorbeigegangen ist. In der Mehrheit der Studien zeigt sich ein Rückgang des Beschäftigungsvolumens, vor allem über die Arbeitszeit. Negative Beschäftigungswirkungen zeigen sich, obwohl der Mindestlohn immer noch in vielen Fällen illegal umgangen wird. Um nicht falsch verstanden zu werden: Wenn es ein Mindestlohngesetz gibt, dann muss es zur Sicherung der Rechtsstaatlichkeit natürlich auch durchgesetzt werden. Man muss sich aber bewusst sein, dass sich die bereits feststellbaren negativen Beschäftigungswirkungen mit einer strikteren Durchsetzung verstärken werden. Dasselbe ist für eine deutliche Erhöhung des Mindestlohns um 25\%, die weit über die allgemeine Lohnentwicklung hinausgeht, zu erwarten. Das gilt auch dann, wenn die Anhebung aller Voraussicht nach zunächst einmal wenige unmittelbar sichtbare negative Folgen haben wird, einerseits weil die steigende Inflation die realen Effekte abmildert, andererseits weil im Zuge der Corona-Krise derzeit ein beachtlicher Ar- 
beitskräftemangel in Branchen auftritt wie der Gastronomie, die besonders stark vom Mindestlohn betroffen sind. Hier hätte es auch ohne Mindestlohnerhöhung erhebliche Lohnanpassungen nach oben gegeben. Die internationale und nationale Studienlage belegt in der Tendenz die Gefahren, die von Mindestlöhnen für die Beschäftigung ausgehen. Wenn Geringverdienende in der Summe den Anschluss an die Mitte nicht verlieren sollen, dann erweist der Mindestlohn innen einen Bärendienst. Hier ist stattdessen der Sozialstaat gefordert, durch Bildungsmöglichkeiten, Beschäftigungsförderung sowie geeignete Anpassungen im Steuer-Transfer-System zu reagieren.

\section{Literatur}

Burauel, P. et al. (2020), The impact of the German minimum wage on individual wages and monthly earnings, Jahrbücher für Nationalökonomie und Statistik, 240(2-3), 201-231.
Caliendo, M. et. al. (2017), The Short-Term Distributional Effects of the German Minimum Wage Reform, IZA Discussion Paper, Nr. 11246.

Dustmann, C. et al. (2021), Reallocation Effects of the Minimum Wage, Quarterly Journal of Economics, im Erscheinen.

Fedorets, A. et al. (2020), Lohnungleichheit in Deutschland sinkt, DIW Wochenbericht, 87(7), 91-97.

Manning, A. (2021), The elusive employment effect of the minimum wage, Journal of Economic Perspectives, 35(1), 3-26.

Mindestlohnkommission (2020), Dritter Bericht zu den Auswirkungen des gesetzlichen Mindestlohns, Bericht der Mindestlohnkommission an die Bundesregierung nach $\S 9$ Abs. 4 Mindestlohngesetz.

Neumark, D. und P. Shirley (2021), Myth or measurement: What does the new minimum wage research say about minimum wages and job loss in the United States?, National Bureau of Economic Research Working Paper, 28388, Januar.

Schröder, C. und C. Kestermann (2020), Mindestlohn und Einkommensarmut, IW-Trends-Vierteljahresschrift zur empirischen Wirtschaftsforschung, 47(2), 107-127.

Statistisches Bundesamt (2020), Verdiensterhebung 2019: Erhebung über die Wirkung des gesetzlichen Mindestlohns auf die Verdienste und Arbeitszeiten der abhängig Beschäftigten, https://www.destatis.de/DE/Themen/Arbeit/Verdienste/Mindestloehne/Publikationen/ Downloads-Mindestloehne/verdiensterhebung-mindestlohn-2019. pdf?_blob=publicationFile (21. November 2021). 